\title{
Not a dilemma: target deconvolution in drug discovery
}

"The perceived failure of target-based drug discovery in current times of "personal genomes' has led to the renaissance of a more holistic approach that might better suit the polygenic and/or multifactorial nature of most diseases."

Worldwide, the requirement for innovative medicines is growing and major common diseases are still faced with a tremendous unmet medical need. While this is more than disappointing from a patient's point of view, at the same time, it represents a huge business opportunity for the pharmaceutical industry. Unfortunately, the industry as a whole is not very successful in making new drugs. In fact, in 2007 only 17 new molecular entities (NMEs) and two biologic license applications were approved by the US FDA, representing a 25 -year low and a continuation of the steady decline observed for many years. Across all therapeutic areas, less than $11 \%$ of new medical entities entering clinical development reach the market. Thus, the 'innovation deficit' also has an enormous economical impact because discovery and development of a new drug typically takes more than 10 years and can cost in excess of US $\$ 800$ million.

Most of the potential drugs - especially those with a new mechanism of action - fail because of a lack of efficacy observed in Phase II clinical trials. One of the many important reasons for this misery is a 'reductionistic' target-based approach for drug discovery, paired with insufficient understanding of the pathophysiology of disease. In the 1990s, this target-based approach largely replaced the more holistic approach of screening compounds for phenotypic changes in complex systems, such as living animals and isolated organs, as it was thought to be more rational and efficient. The sequencing of the human genome, which was in progress at that time and heralded the advent of a new era with all potential drug targets known, greatly spurred on this approach. In fact, all major pharma and many biotech companies wanted to be part of the 'gold rush' and tried to stake their claim in it, which is also reflected by hundreds of gene patents filed during that period of time.
The perceived failure of target-based drug discovery in current times of 'personal genomes' has led to the renaissance of a more holistic approach that might better suit the polygenic and/or multifactorial nature of most diseases. Here, responses of entire systems are measured by screening compounds for phenotypic effects and no a priori selection of molecular targets is necessary. As an analogy to classical forward genetic screens in model organisms, this approach is also referred to as 'chemical genetics'. While many model systems, such as the worm Caenorhabditis elegans, the fly Drosophila melanogaster, the zebrafish Dario reo and the mouse Mus musculus, can be employed for drug screening, mainly mammalian cells are currently compatible with the needs of high throughput screening (HTS) with a phenotypic read-out. Such cells can be isolated from organs and kept in culture for a limited period of time (primary cell cultures) or can be immortalized, resulting in permanent cell lines. For HTS, permanent cell lines are mainly utilized as they are much easier to manipulate, can be grown to large numbers and many are commercially available. Moreover, these cell lines can be more easily genetically engineered to mimic aspects of disease or to monitor the modulation of disease-relevant pathways and mechanisms.

Typical phenotypic read-outs are represented by biochemical assays specific to the effect under investigation, such as the measurement of cell death using fluorescent cell-viability dyes and reporter gene assays monitoring the modulation of the pathways under investigation. With advances in cellular imaging technologies, many different phenotypic changes including morphological changes (e.g., cell shape, neurite outgrowth and proliferation) can now be measured simultaneously in a plate-based format by employing high-content analysis systems. These systems

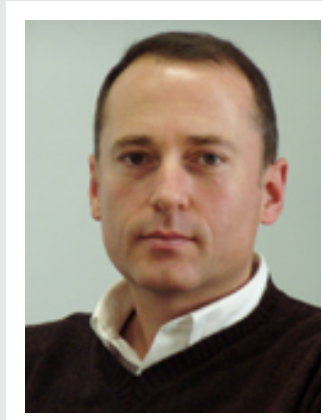

Georg C Terstappen Siena Biotech S.p.A., Strada del Petriccio e Belriguardo 35, 53100 Siena, Italy

Tel.: +39057 7381 302

Fax: +39057 7381 208

E-mail: gterstappen@

sienabiotech.com 
combine automated microscopy and automated image analysis and represent a powerful tool for phenotypic analysis.

Whatever combination of disease model and read-out measure might have been used for the identification of active compounds, the retrospective identification of the molecular target(s) underlying the observed phenotypic responses (termed target deconvolution) is important. Knowledge of the molecular target(s) will aid understanding of the (patho)mechanism(s) of the disease, help rational drug design and enable efficient structure-activity relationship (SAR) studies during chemical optimization as target-based assays can be developed. Moreover, aspects related to target-specific toxicity and potential side effects can be investigated, thus helping to avoid later-stage 'attrition' early on. Many different technologies exist that can be employed to identify the molecular target(s). Selection of the method-of-choice, which needs to be decided on a case-by-case basis, is often primarily governed by the properties of the active molecule. Assuming that physical interaction is a prerequisite for the observed functional effects, methods leading to direct identification of targets typically exploit affinity between the active molecule and its target protein.

'Classical' affinity chromatography-based techniques have been successfully employed for target deconvolution and a significant advantage of these methods is that target proteins maintain their 3D structure, including posttranslational modifications. Strategies based on protein microarrays represent an alternative to affinity chromatography. Identification of proteins expressed at low levels in vivo is not a priori compromized, since large numbers of individually purified proteins are spotted in equal amounts to generate the microarray. Protein microarrays are closed systems and, thus, only those proteins that have been immobilized on the microchip can be identified. A method referred to as reverse-transfected microarrays utilizes microarrays of cells expressing defined cDNAs, thus circumventing the requirement to use large numbers of individually purified proteins. Expression cloning-based methods, such as mRNA display, phage display and three hybrid systems, all include an affinity step as well. As proteins are expressed from fusion constructs of cDNA libraries, the properties of these recombinant proteins might differ from their native counterparts, in particular in terms of post-translational modifications. In addition, access to advanced molecular biology skills is needed, which is not the case for affinity chromatography. A disadvantage of all direct methods for target deconvolution is represented by the need to chemically modify the active molecules for the purposes of labeling and/or immobilization. This implies that access to synthetic chemistry is necessary and, often, even a limited SAR study will have to be performed in order to identify those positions in the molecule that can be modified without compromising its biological activity.

Application of a number of technologies, such as comprehensive DNA microarray or proteomic techniques, which do not need modification of the chemical molecules, can also in a more indirect way lead to the identification of the molecular targets. For instance, the analysis of global geneexpression changes of an organism mediated by an active molecule and measured by DNA microarray technology can aid understanding of the molecule's mechanism of action (MoA). In the form of 'connectivity maps', these results can be used to connect chemical molecules, genes and disease in a systematic fashion. Studying global changes in the protein constitution of an organism in response to exposure to an active molecule by proteomics technologies allows the generation of a 'proteomic compound signature', which might also help in understanding the chemical molecule's MoA if it is compared with signatures obtained from known reference compounds.

\section{"Target deconvolution is complex but not a dilemma in the true sense of this Greek word because many different technologies exist that have successfully led to the identification of molecular targets underlying a drug's action."}

The final goal of all target-deconvolution strategies is the identification of biological molecules that directly interact with the active molecule, as well as confirmation that modulation of the identified biological target causes functional effects in the phenotypic assay system that was initially used for the identification of the chemical molecule. A number of methods can be used to confirm physical interaction (e.g., surface plasmon resonance) and associated phenotypic changes can be verified by functional studies employing RNA interference - leading to target gene knock-down - or gene overexpression. Transgenic and knock-out animals can be generated for detailed studies of phenotype in vivo. 
"As timelines might be long and outcomes unpredictable, it might be comforting to know that for some of the approved drugs on the market, the molecular target(s) are still, as of today, unknown."

Target deconvolution is complex but not a dilemma in the true sense of this Greek word because many different technologies exist that have successfully led to the identification of molecular targets underlying a drug's action. For instance, cytosolic malate dehydrogenase was identified as a target of the anticancer drug E7070 and glyoxalase 1 as a target of the antiinflammatory drug indomethacin. Critical considerations are the choice of the most appropriate method and deciding when the right time is to start deconvolution activities during the progression of active chemical molecules through the drug-discovery process. As timelines might be long and outcomes unpredictable, it may be comforting to know that for some of the approved drugs on the market, the molecular target(s) are still, as of today, unknown.

\section{Financial \& competing interests disclosure}

The author has no relevant affliations or financial involvement with any organization or entity with a financial interest in or financial conflict with the subject matter or materials discussed in the manuscript. This includes employment, consultancies, honoraria, stock ownership or options, expert testimony, grants or patents received or pending, or royalties.

No writing assistance was utilized in the production of this manuscript. 